\title{
Local and Locoregional Disease Free Survival in Patients Receiving NSM or SSM Compared with Conventional Mastectomy
}

\author{
Zachary H. Hopkins ${ }^{1}$, Jonathan Frandsen², Katherine E. Poruk ${ }^{3}$, Jayant Agarwal ${ }^{4}$, Matthew Poppe ${ }^{2 *}$ \\ ${ }^{1}$ University of Utah School of Medicine, Salt Lake City, UT, USA \\ ${ }^{2}$ Department of Radiation Oncology, Huntsman Cancer Hospital, University of Utah School of Medicine, Salt Lake City, UT, USA \\ ${ }^{3}$ Department of Surgery, The Johns Hopkins Hospital, Baltimore, MD, USA \\ ${ }^{4}$ Division of Plastic and Reconstructive Surgery, University of Utah School of Medicine, Salt Lake City, UT, USA
}

*Corresponding author: Matthew Poppe, MD, University of Utah Huntsman Cancer Hospital, Department of Radiation Oncology, 1950 Circle of Hope Room 1570, Salt Lake City UT 84112, USA, Tel: 801-581-2396/ Fax: 801-585-3502;

E-mail: Matthew.poppe@hci.utah.edu

\begin{abstract}
Introduction: In this study we evaluated local recurrence-free survival (LRFS) and locoregional recurrence-free survival (LRRFS) for women at our institution who received NSM and SSM as compared to patients receiving traditional non-skin or nipple sparing mastectomies (TM).

Methods: From 2005 to 2014, women with T1-2, N0, M0 disease who did not receive radiation were included in the analysis. Patients were separated into one of three cohorts based on mastectomy type. Kaplan Meier survival estimates were used to estimate LRFS and LRRFS. Cox proportional hazards analysis was used to calculate risk factors contributing to these outcomes.

Results: At eight years, LRFS was $95.9 \%$ for TM, 100.0\% for NSM and $96.8 \%$ for SSM. Log-rank analysis showed no significant difference in LRFS between the 3 groups $(\mathrm{p}=0.67)$. At eight years LRRFS was $94.3 \%$ for TM, 92.6\% for NSM and $91.4 \%$ for SSM with no significant differences seen among these groups $(p=0.51)$. In univariate analyses, only T-stage was a significant risk factor for local recurrence (HR 3.84, 95\% CI $1.17-12.6, \mathrm{p}=0.03$ ).

Conclusions: For this patient population, SSM and NSM appear to be safe and equivalent to TM at 8 years of follow up.
\end{abstract}

Received Date: November 9, 2016

Accepted Date: February 28, 2017

Published Date: March 07, 2017

Citation: Hopkins, Z.H., et al. Local and Locoregional Disease Free Survival in Patients Receiving NSM or SSM Compared with Conventional Mastectomy. (2017) Int J Cancer Oncol 4(2): 207- 212.

DOI: $10.15436 / 2377-0902.17 .1213$

Keywords: Mastectomy; Skin -sparing mastectomy (SSM); Nipple-sparing mastectomy (NSM); Breast cancer

\section{Introduction}

Mastectomy has been a mainstay surgical technique for breast cancer treatment for over a century. Traditionally, the original surgical technique pioneered by William Halsted included removal of the breast, overlying skin, underlying chest muscles (including pectoralis major and pectoralis minor), and lymph nodes of the axilla ${ }^{[1]}$. In the last $10-20$ years, mastectomies have become more conservative to preserve both function and cosmesis ${ }^{[2,3]}$. Today, pectoralis muscles are rarely removed and sentinel node procedures are often considered sufficient $t^{[4]}$. Over the last 15 years, techniques to improve cosmetic outcomes by preserving the skin and nipple overlying the breast have been pioneered. These techniques include: skin-sparing mastectomy (SSM) where the breast tissue and the nipple areolar complex (NAC) are removed but the skin overlying the breast is preserved, and the nipple sparing mastectomy (NSM), where the skin and NAC are preserved ${ }^{[3,5-8]}$. Preserving the skin in an SSM and the preservation of the NAC in the NSM results in better cosmesis, improved patient satisfaction, and quality of life ${ }^{[3,5,8,9]}$.

Although an increasing number of women are receiving these skin and nipple-conserving therapies, evidence for these procedures' oncologic safety is still evolving. Women with node negative, T1-T2 breast cancers do not routinely receive post-mastectomy radiation, given the low potential of local or locoregional recurrence. In women undergoing an SSM or NSM, 
a small amount of breast tissue is likely left behind, potentially placing these women at a higher risk for a local recurrence ${ }^{[10,11]}$. The goal of this study was to evaluate local and locoregional recurrence-free survival in women at our institution who received NSM and SSM compared to patients receiving a traditional nonskin or nipple sparing mastectomy (TM).

\section{Methods}

We utilized a tumor registry that included all women treated for breast cancer within the University of Utah Healthcare system. This registry contains prospectively collected data over the last four decades, including the use of NSM and SSM. With Institutional Review Board approval, the data from the registry was analyzed.

Women were identified in the database with breast cancer treated from 2005, the time at which skin-sparing mastectomies became utilized at this institution, until 2014. Only women who underwent mastectomy and were found to have a primary tumor $<5 \mathrm{~cm}$ and negative lymph nodes were included. Women who received adjuvant radiation were excluded. For this study, traditional mastectomy (TM) was defined as any mastectomy that was not a SSM or NSM. This included: total mastectomy, modified radical mastectomy, and radical mastectomy. Women with incomplete staging, metastatic disease, in-situ only histology, and prophylactic mastectomies were excluded from the analysis. Local recurrence (LR) was defined as any recurrence along the chest wall, and locoregional recurrence (LRR) was defined as any recurrence along the chest wall or regional draining lymphatics, including cases when LRR was diagnosed concurrently with a distant metastasis.
Women were grouped into three cohorts: (1) women who underwent a TM, (2) women who received NSM, and (3) women who received SSM. Chi-square analyses were employed for comparisons of categorical variables in the three groups. Kruskal-Wallis and ANOVA were used for continuous variables. Two-tailed tests were used for all testing with a p-value of $<0.05$ considered significant. Kaplan-Meier tests were used to analyze our primary endpoints which included local recurrence-free survival (LRFS) and locoregional recurrence-free survival (LRRFS). Cox proportional hazards models were used to test association of risk factors with LRFS and LRRFS. All statistical analyses were performed with $\mathrm{R}$, version 3.2.0.

\section{Results}

Four hundred seventy patients were identified who met our inclusion and exclusion criteria. Of these patients, 402 $(85.5 \%)$ were treated with TM, $31(6.6 \%)$ with nipple-sparing mastectomy (NSM), and $37(7.9 \%)$ were treated with skin-sparing mastectomy (SSM). Median follow-up for all cohorts was 4.94 years. Median follow-up for individual cohorts were 5 years for TM, 5 years for NSM and 6 years for SSM. Patient and tumor characteristics are given in Table 1 . Of note, patients receiving NSM were younger (median age of diagnosis was 48 compared to 56 for TM and 57 for SSM $p=<0.03$ ). Mean tumor size tended to be smaller in NSM patients $(1.16 \mathrm{~cm}$ compared with $1.48 \mathrm{~cm}$ for SSM and $1.71 \mathrm{~cm}$ for TM, $\mathrm{p}=0.03$ ). There were no significant differences in T-stage, multifocality, lymphovascular space invasion (LVSI), grade, margin status, race, tobacco usage, use of hormone or chemotherapy, hormone receptor status, and human epidermal growth factor (HER2) receptor status between the 3 groups (see Table 1).

Table 1: Patient and Tumor Characteristics.

\begin{tabular}{|c|c|c|c|c|}
\hline Variable & $T M(n=402)$ & $\operatorname{NSM}(n=31)$ & $\operatorname{SSM}(n=37)$ & p-value \\
\hline Median Age at Dx $\left(25-75^{\text {th }}\right.$ percentile $)$ & $56(48-66)$ & $48(43-55)$ & $57(48-67)$ & $0.004 \dagger$ \\
\hline Mean Tumor Size (sd) & $1.71(1.12)$ & $1.16(0.62)$ & $1.48(1.10)$ & $0.025 \dagger \dagger$ \\
\hline Median Follow-Up Time $\left(25-75^{\text {th }}\right.$ percentile $)$ & $5(3.2-7.0)$ & $5(4.0-6.0)$ & $6(4.8-8)$ & $<0.25 \dagger$ \\
\hline T-Stage $(\%)$ & & & & $0.09 \S$ \\
\hline T1 & $291(72.4)$ & $28(90.3)$ & $27(73.0)$ & \\
\hline T2 & $111(27.6)$ & $3(9.7)$ & $10(27.0)$ & \\
\hline Multifocal (\%) & & & & 0.99 \\
\hline No & $244(79.0)$ & $24(80.0)$ & $29(78.4)$ & \\
\hline Yes & $65(21.0)$ & $6(20.0)$ & $8(21.6)$ & \\
\hline LVSI & & & & 0.09 \\
\hline No & $227(87.3)$ & $27(96.4)$ & $35(97.2)$ & \\
\hline Yes & $33(12.7)$ & $1(3.6)$ & $1(2.8)$ & \\
\hline Tumor Grade (\%) & & & & 0.43 \\
\hline I & $83(22.1)$ & $10(33.3)$ & $7(19.4)$ & \\
\hline II & $167(44.5)$ & $11(36.7)$ & $20(55.6)$ & \\
\hline III & $125(33.3)$ & $9(30.0)$ & $9(25.0)$ & \\
\hline Margin Status (\%) & & & & 0.07 \\
\hline Negative & $162(60.4)$ & $24(82.8)$ & $26(72.2)$ & \\
\hline Close & $79(29.5)$ & $5(17.2)$ & $7(19.4)$ & \\
\hline Positive & $27(10.1)$ & $0(0.0)$ & $3(8.3)$ & \\
\hline Estrogen Receptor & & & & 0.62 \\
\hline
\end{tabular}


Comparison of NSM and SSM patience with Conventional Mastectomy

OMMEgA Publishers

\begin{tabular}{|l|c|c|c|c|}
\hline No & $84(21.4)$ & $8(25.8)$ & $6(16.2)$ & \\
\hline Yes & $125(78.6)$ & $23(74.2)$ & $31(83.8)$ & \\
\hline Progesterone Receptor & & & & 0.52 \\
\hline No & $107(27.6)$ & $8(25.8)$ & $7(18.9)$ & \\
\hline Yes & $280(72.4)$ & $23(74.2)$ & $30(81.1)$ & \\
\hline HER2 Receptor & & & & 0.76 \\
\hline No & $133(84.7)$ & $23(79.3)$ & $29(82.9)$ & \\
\hline Yes & $24(15.3)$ & $6(20.7)$ & $6(17.1)$ & \\
\hline Chemotherapy (\%) & & & & 0.70 \\
\hline No & $254(65.5)$ & $22(71.0)$ & $24(66.7)$ & \\
\hline Yes & $134(34.5)$ & $9(29.0)$ & $12(33.3)$ & \\
\hline Hormone Therapy (\%) & & & & 0.53 \\
\hline No & $153(38.2)$ & $9(29.0)$ & $15(41.7)$ & \\
\hline Yes & $248(61.8)$ & $22(71.0)$ & $21(58.3)$ & \\
\hline Ethinicity (\%) & & & & 0.56 \\
\hline White/Caucasian & $377(93.8)$ & $29(93.5)$ & $33(89.2)$ & \\
\hline Other & $25(6.2)$ & $2(6.5)$ & $4(10.8)$ & \\
\hline Tobacco (\%) & & & & 0.17 \\
\hline No & $220(69.8)$ & $24(85.7)$ & $23(65.7)$ & \\
\hline Yes & $95(30.2)$ & $4(14.3)$ & $12(34.3)$ & \\
\hline
\end{tabular}

Abbreviations: TM: Traditional Mastectomy; NSM: Nipple Sparing Mastectormy; SSM: Skin Sparing Mastectomy

Data in parenthesis are percentages. $\uparrow$ Computed from Kruskal-Wallis. $\dagger \dagger$ Computed from ANOVA, $\S$ Computed from $\mathrm{X}^{2}$

Among patients treated with TM, ten local recurrences $(2.5 \%)$ were seen and seventeen locoregional recurrences $(4.2 \%)$ occurred. Among patients treated with NSM, no local recurrences were seen and two locoregional recurrences $(6.5 \%)$ occurred. For patients treated with SSM one local recurrence $(2.7 \%)$ was seen and four locoregional recurrences $(10.8 \%)$ occurred over the 8 year follow-up period (Table 2). Of note, none of the recurrences found in the NSM group occurred in the nipple areolar complex.

Additionally, no difference was seen in overall survival by log-rank test $(\mathrm{p}=0.54)$.

Table 2: Recurrences.

\begin{tabular}{|c|l|l|l|l|}
\hline & TM (\%) & NSM (\%) & SSM (\%) & p-value \\
\hline Local Recurrence & & & & $0.67 \dagger$ \\
\hline No & $385(97.5)$ & $31(100.0)$ & $36(97.3)$ & \\
\hline Yes & $10(2.5)$ & $0(0.0)$ & $1(2.7)$ & \\
\hline $\begin{array}{l}\text { Locoregional Re- } \\
\text { currence }\end{array}$ & & & & 0.50 \\
\hline No & $385(95.8)$ & $29(93.5)$ & $33(89.2)$ & \\
\hline Yes & $17(4.2)$ & $* 2(6.5)$ & $4(10.8)$ & \\
\hline $\begin{array}{l}\text { Distant Recur- } \\
\text { rence }\end{array}$ & & & & 0.43 \\
\hline No & $384(95.5)$ & $31(100.0)$ & $36(97.3)$ & \\
\hline Yes & $18(4.5)$ & $0(0.0)$ & $1(2.7)$ & \\
\hline
\end{tabular}

Abbreviations: TM: Traditional Mastectomy; NSM: Nipple Sparing Mastectormy; SSM: Skin Sparing Mastectomy

Number in parentheses is percentages.

$\dagger$ Calculated with Chi-Squared test.

* No LRR within the NSM cohort occurred within the nipple areolar complex.

\section{Five and Eight Year Recurrence Free Survival}

At five years, LRFS was $97.4 \%$ for TM, $100.0 \%$ for NSM and $96.8 \%$ for SSM (Figure 1). At five years, LRRFS was: $96.9 \%$ for TM, $92.6 \%$ for NSM, and $91.6 \%$ for SSM, with no significant differences seen $(\mathrm{p}=0.60)$ (Figure 2).

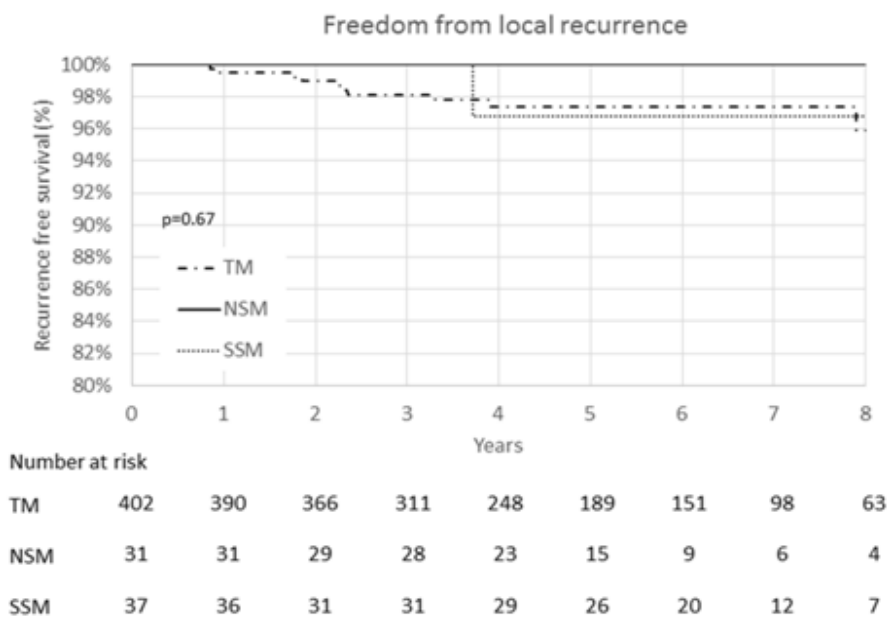

Figure 1: Kaplan-Meier curve of local recurrence-free survival. No significant differences in local recurrence-free survival were seen between traditional mastectomy (TM), nipple sparing mastectomy (NSM), or skin sparing mastectomy (SSM).

At eight years, LRFS was $95.9 \%$ for TM, $100.0 \%$ for NSM and $96.8 \%$ for SSM (Figure 1). The log-rank test shows no significant differences between these 3 groups $(p=0.67)$. At eight years, LRRFS was: $94.3 \%$ for TM, $92.6 \%$ for NSM, and $91.4 \%$ for SSM, again with no significant differences seen ( $\mathrm{p}=$ 0.51 ) (Figure 2). When grouping NSM and SSM together, there was still no difference in LRFS or LRRFS ( $p=0.87$ and $p=0.10$ 
Comparison of NSM and SSM patience with Conventional Mastectomy respectively). Additionally, there were no differences in eightyear distant recurrence free survival or recurrence free survival when comparing TM versus SSM $/ \mathrm{NSM}(\mathrm{p}=0.58$ and $\mathrm{p}=0.26$, respectively).

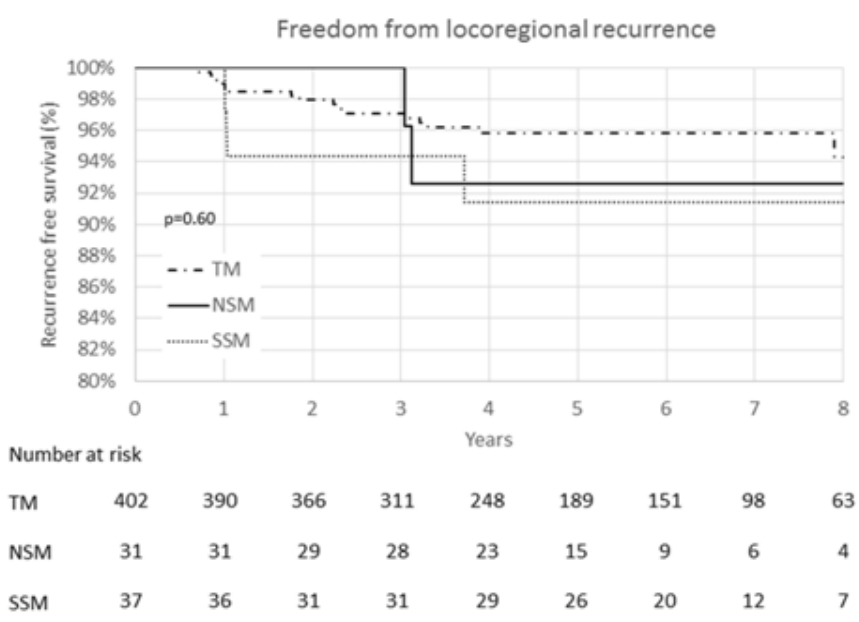

Figure 2: Kaplan-Meier curve of locoregional recurrence-free survival. No significant differences in locoregional recurrence-free survival were seen between traditional mastectomy (TM), nipple sparing mastectomy (NSM), or skin sparing mastectomy (SSM).

\section{Univariate Analysis for Local and Locoregional Recur- rence-Free Survival}

As no local recurrences were observed among women undergoing NSM, these women were combined with the SSM cohort for Cox proportional hazards analysis of recurrence-free survival. In univariate analysis, tumor T-stage was found to be predictive of LR (HR 3.84, 95\% CI $1.17-12.6, \mathrm{p}=0.03$ ) and higher grade was found to be slightly protective (HR $0.22,95 \%$ CI $0.05-0.90, p=0.03$ for grade II and HR $0.11,95 \%$ CI 0.01 $0.91, \mathrm{p}=0.04$ for grade III) (Table 3). No risk factor was significantly associated with an increased risk of LRR, although receipt of hormone therapy was protective (HR $0.36,95 \%$ CI $0.15-0.87, p=0.02$ ) (Table 4). Type of mastectomy was not significantly associated with an increased risk of LR (HR 0.56, 95\% CI $0.07-4.41, \mathrm{p}=0.0 .59$ ) or LRR (HR 1.77, 95\% CI $0.65-4.82$, $\mathrm{p}=0.27$ ). Missing values in some of the subgroups prevented estimation of the interaction between T-stage and mastectomy type for LR. Analysis for interaction of significant variables with type of mastectomy was performed. No interaction was seen between receipt of hormone therapy and mastectomy type for LRR $(p=0.54)$ and the protective effect of hormone therapy became non-significant when controlling for mastectomy type $(\mathrm{p}=0.07)$.

Table 3: Cox Proportional Hazard Analyses for Local Disease Free Survival.

\begin{tabular}{|l|l|l|l|}
\hline & HR & 95\% CI & p-value \\
\hline Age at Diagnosis & 1.02 & $0.97-1.07$ & 0.42 \\
\hline Tumor Size & 1.26 & $1.07-1.64$ & 0.08 \\
\hline Mastectomy Type & 1 & & \\
\hline TM & 0.57 & $0.07-4.41$ & 0.59 \\
\hline NSM/SSM & 1 & & \\
\hline T-Stage & 1 & \\
\hline T1 &
\end{tabular}

\begin{tabular}{|c|c|c|c|}
\hline $\mathrm{T} 2$ & 3.84 & $1.17-12.6$ & 0.03 \\
\hline \multicolumn{4}{|l|}{ Multifocality } \\
\hline No & 1 & & \\
\hline Yes & 1.26 & $0.26-6.27$ & 0.78 \\
\hline \multicolumn{4}{|l|}{ Tumor Grade } \\
\hline I & 1 & & \\
\hline II & 0.22 & $0.05-0.90$ & 0.03 \\
\hline III & 0.11 & $0.01-0.91$ & 0.04 \\
\hline \multicolumn{4}{|l|}{ LVSI } \\
\hline No & 1 & & \\
\hline Yes & 2.44 & $0.27-21.9$ & 0.43 \\
\hline \multicolumn{4}{|l|}{ ER } \\
\hline No & 1 & & \\
\hline Yes & 2.37 & $0.30-18.71$ & 0.42 \\
\hline \multicolumn{4}{|l|}{ PR } \\
\hline No & 1 & & \\
\hline Yes & 1.26 & $0.26-6.12$ & 0.77 \\
\hline \multicolumn{4}{|l|}{ Chemotherapy } \\
\hline No & 1 & & \\
\hline Yes & 1.83 & $0.53-6.32$ & 0.34 \\
\hline \multicolumn{4}{|l|}{ Hormone Therapy } \\
\hline No & 1 & & \\
\hline Yes & 0.58 & $0.17-2.01$ & 0.39 \\
\hline \multicolumn{4}{|l|}{ Tobacco Usage } \\
\hline No & 1 & & \\
\hline Yes & 0.75 & $0.15-6.74$ & 0.73 \\
\hline \multicolumn{4}{|l|}{ Race } \\
\hline White/Caucasian & 1 & & \\
\hline Other & 1.52 & $0.19-11.9$ & 0.69 \\
\hline
\end{tabular}

Abbreviations: TM: Traditional Mastectomy; NSM: Nipple Sparing Mastectormy; SSM: Skin Sparing Mastectomy

All analyses were univariate. HER2 receptor status and carcinoma margins were not formally tested because no observations were present in 1 or more subgroups.

Table 4: Cox Proportional Hazard Analyses for Locoregional Disease Free Survival.

\begin{tabular}{|c|c|c|c|}
\hline & HR $(95 \%$ CI $)$ & $95 \% \mathrm{CI}$ & P-value \\
\hline Age at Diagnosis & 1.02 & $0.99-1.06$ & 0.14 \\
\hline Tumor Size & 1.15 & $0.88-1.50$ & 0.30 \\
\hline \multicolumn{4}{|l|}{ Mastectomy Type } \\
\hline $\mathrm{TM}$ & 1 & & \\
\hline NSM/SSN & 1.77 & $0.65-4.82$ & 0.27 \\
\hline \multicolumn{4}{|l|}{ T-Stage } \\
\hline $\mathrm{T} 1$ & 1 & & \\
\hline $\mathrm{T} 2$ & 1.73 & $0.72-4.13$ & 0.22 \\
\hline \multicolumn{4}{|l|}{ LVSI } \\
\hline No & 1 & & \\
\hline Yes & 1.16 & $0.26-5.19$ & 0.85 \\
\hline \multicolumn{4}{|l|}{ Multifocal } \\
\hline No & 1 & & \\
\hline
\end{tabular}




\begin{tabular}{|c|c|c|c|}
\hline Yes & 0.87 & $0.25-3.07$ & 0.83 \\
\hline \multicolumn{4}{|l|}{ Tumor Grade } \\
\hline I & 1 & & \\
\hline II & 0.46 & $0.17-1.23$ & 0.12 \\
\hline III & 0.42 & $0.13-1.28$ & 0.13 \\
\hline \multicolumn{4}{|c|}{ Surgical Margins } \\
\hline Negative & 1 & & \\
\hline Close & 0.46 & $0.10-2.12$ & 0.32 \\
\hline Positive & 2.15 & $0.65-7.06$ & 0.21 \\
\hline \multicolumn{4}{|l|}{ ER } \\
\hline No & 1 & & \\
\hline Yes & 1.15 & $0.38-3.41$ & 0.81 \\
\hline \multicolumn{4}{|l|}{ PR } \\
\hline No & 1 & & \\
\hline Yes & 1.02 & $0.37-2.83$ & 0.98 \\
\hline \multicolumn{4}{|c|}{ Chemotherapy } \\
\hline No & 1 & & \\
\hline Yes & 1.35 & $0.57-3.21$ & 0.50 \\
\hline \multicolumn{4}{|c|}{ Hormone Therapy } \\
\hline No & 1 & & \\
\hline Yes & 0.36 & $0.15-0.87$ & 0.02 \\
\hline \multicolumn{4}{|c|}{ Tobacco Usage } \\
\hline No & 1 & & \\
\hline Yes & 0.83 & $0.30-2.30$ & 0.71 \\
\hline \multicolumn{4}{|l|}{ Race } \\
\hline No & 1 & & \\
\hline Yes & 1.49 & $0.35-6.37$ & 0.59 \\
\hline
\end{tabular}

Abbreviations: TM: Traditional Mastectomy; NSM: Nipple Sparing Mastectormy; SSM: Skin Sparing Mastectomy LVSI: Lymphovascular Space Invasion; ER: Estrogen Receptors; PR: Progesterone Receptors. HER2 had no events in one of the subgroups and therefore was not tested.

\section{Discussion}

In this study we set out to compare LR and LRR in women undergoing SSM and NSM compared to a TM. We specifically wanted to analyze women who were not typical candidates for post-mastectomy radiation therapy. We defined this population as women with node negative, T1-T2 breast cancers with no metastasis. We found no increased risk of local or locoregional failure with SSM or NSM compared to TM in this low risk population. Additionally, we found no decrease in overall survival in patients receiving NSM and SSM compared to those receiving TM.

While numerous studies exist evaluating the oncological safety of SSM, NSM, and TM, these studies evaluate each technique in isolation ${ }^{[7,9,12-18]}$ or SSM compared to $\mathrm{NSM}^{[2,3,19]}$. To our knowledge no other studies have compared rates of LR and LRR among these three surgical techniques with as large a sample size and duration of follow-up as was assessed in our study. Of note, NSM and SSM recurrence rates seen among our patients are similar ${ }^{[2,12,14,19]}$ or lower ${ }^{[15,17,19,20]}$ compared to rates reported previously. A recent 2015 meta-analysis using patient information from 19 studies evaluating NSM found the mean

LR rate at a follow-up time of 5 or more years to be $8.2 \%{ }^{[15]}$. A large Japanese study showed a long term LR rate of $8.2 \%$ for NSM and $7.2 \%$ for TM with no significant difference between the $\mathrm{two}^{[21]}$. Our recurrence rates were similar or lower. However, some of these studies included patients with stage 0-III disease, positive nodal status, and/or patients receiving radiation therapy $^{[2,13,14,18,19,20]}$. Only one other study included only women who did not receive radiation and reported a $20.8 \%$ locoregional recurrence rate in women receiving a NSM. This study included women with higher risk disease and stage I-III cancers, making a direct comparison with our study difficult.

In our NSM cohort there were no recurrences in the nipple areolar complex. This is important as the terminal-duct lobular units that remain in the conserved nipple areolar complex potentially hold occult cancer remnants ${ }^{[11]}$. Our study suggests that recurrence in the nipple areolar complex is rare, at least in women with already low risk disease. More studies are needed to better establish the risk for local recurrence to the nipple areolar complex.

Our study has limitations. First, as a retrospective study there may be biases which could not be adequately accounted for; due to the relatively low recurrence rates observed in our patients, our ability to perform multivariate analyses was limited. Nevertheless, we did find T-stage to be significantly associated with LR (HR 3.84, 95\% CI 1.17 - 12.6, p = 0.03). Grade was also significant, but as a protective factor (HR $0.22,95 \% \mathrm{CI} 0.05$ $-0.90, p=0.03$ for grade II and HR $0.11,95 \%$ CI $0.01-0.91$, $\mathrm{p}=0.04$ for grade III). No significant associations were seen for increased risk of LRR, although receipt of hormone therapy was significant for being protective against recurrence (HR 0.36 , $95 \%$ CI $0.15-0.87, \mathrm{p}=0.03$ ). Both tumor size and hormone therapy were similarly found to be significant risk factors in a large meta-analysis of nipple involvement in $\mathrm{NSM}^{[10]}$. Similar results were seen for any recurrence ${ }^{[10,11,18]}$. These studies suggested that tumor location (central or peripheral), tumor size, multifocality, tumor grade, presence of ductal carcinoma in situ (DCIS), LVSI, negative estrogen-receptor (ER) status and positive HER2 status were all predictive of recurrence. While our study did not demonstrate these as being significant it may have been due to our selection of patients with low risk disease, and the resultant low number of recurrences. Also, our finding of a higher grade being protective was unexpected and is contrary to evidence in the literature ${ }^{[10,11,18]}$. We suspect that this is the result of sampling, or an unforeseen confounding and does not represent a true finding. We did not have access to cause of death so cause-specific survival could not be determined. Finally, since NSM and SSM are recently developed surgical techniques the number of patients available for analysis, with up to 8 years of follow-up, was limited. More studies are needed to better define the long-term risk of LR and LRR.

This study provides interesting, novel insight regarding LRR rates in women undergoing NSM and SSM compared to TM. The goal of the study was to compare rates of LR and LRR in women not typically considered candidates for post-mastectomy radiotherapy. Importantly, we found no increased risk of relapse in the skin or nipple sparing procedures as compared to traditional mastectomy. 


\section{Conclusion}

In conclusion, in women with low risk breast cancer receiving no adjuvant radiation, the 8-year rates of LR or LRR are similar when comparing TM versus SSM or NSM. For this early stage population, we found both NSM and SSM to be safe surgical alternatives to TM with the added benefit of better cosmesis and possible better patient satisfaction. More studies are needed to identify long-term risk and better identify risk factors for recurrence.

Conflicts of Interest: All authors report no conflicts of interest.

\section{References}

1. Ghossain, A., Ghossain, M.A. History of mastectomy before and after Halsted. (2009) J Méd Liban 57(2): 65-71.

2. Yi, M., Kronowitz, S.J., Meric-Bernstam, F., et al. Local, region$\mathrm{al}$, and systemic recurrence rates in patients undergoing skin-sparing mastectomy compared with conventional mastectomy. (2011) Cancer 117(5): 916-924.

3. Poruk, K.E., Ying, J., Chidester, J.R., et al. Breast cancer recurrence after nipple-sparing mastectomy: one institution's experience. (2015) Am J Surg 209(1): 212-217.

4. Donker, M., van Tienhoven, G., Strayer, M.E., et al. Radiotherapy or surgery of the axilla after a positive sentinel node in breast cancer (EORTC 10981-22023 AMAROS): a randomised, multicentre, open-label, phase 3 non-inferiority trial. (2014) Lancet Oncol 15(12): 1303-1310. 5. Petit, J.Y., Veronesi, U., Luini, A., et al. When mastectomy becomes inevitable: The nipple-sparing approach. (2005) Breast 14(6): 527-531. 6. Serra, R., Miglietta, A.M., Abonante, S., et al. Skin-Sparing Mastectomy with Immediate Breast and Nipple Reconstruction: A New Technique of Nipple Reconstruction. (2013) Plast Surg Int 2013: 1-5.

7. Carlson, G.W., Bostwick, J., 3rd, Styblo, T.M., et al. Skin-sparing mastectomy. Oncologic and reconstructive considerations. (1997) Ann Surg 225(5): 570.

8. Sacchini, V., Pinotti, J.A., Barros, A.C., et al. Nipple-Sparing Mastectomy for Breast Cancer and Risk Reduction: Oncologic or Technical Problem? (2006) J Am Coll Surg 203(5): 704-714.

9. Patani, N., Mokbel, K. Oncological and aesthetic considerations of skin-sparing mastectomy. (2008) Breast Cancer Res Treat 111(3): 391-403.

10. Zhang, H., Li, Y., Moran, M.S., et al. Predictive factors of nipple involvement in breast cancer: a systematic review and meta-analysis. (2015) Breast Cancer Res Treat 151(2): 239-249.
11. Eisenberg, R.E.K., Chan, J.S.Y., Swistel, A.J., et al. Pathological Evaluation of Nipple-Sparing Mastectomies with Emphasis on Occult Nipple Involvement: The Weill-Cornell Experience with 325 Cases. (2014) Breast J 20(1): 15-21.

12. Endara, M., Chen, D., Verma, K., et al. Breast Reconstruction following Nipple-Sparing Mastectomy: A Systematic Review of the Literature with Pooled Analysis. (2013) Plast Reconstr Surg 132(5): 1043-1054.

13. Medina-Franco, H., Vasconez, L.O., Fix, R.J., et al. Factors associated with local recurrence after skin-sparing mastectomy and immediate breast reconstruction for invasive breast cancer. (2002) Ann Surg 235(6): 814.

14. Caruso, F., Ferrara, M., Castiglione, G., et al. Nipple sparing subcutaneous mastectomy: Sixty-six months follow-up. (2006) Eur J Surg Oncol 32(9): 937-940.

15. de La Cruz, L., Moody, A.M., Tappy, E.E., et al. Overall Survival, Disease-Free Survival, Local Recurrence, and Nipple-Areolar Recurrence in the Setting of Nipple-Sparing Mastectomy: A Meta-Analysis and Systematic Review. (2015) Ann Surg Oncol 22(10): 3241-3249.

16. Downes, K.J., Glatt, B.S., Kanchwala, et al. Skin-sparing mastectomy and immediate reconstruction is an acceptable treatment option for patients with high-risk breast carcinoma. (2005) Cancer 103(5): 906-913.

17. Benediktsson, K.P., Perbeck, L. Survival in breast cancer after nipple-sparing subcutaneous mastectomy and immediate reconstruction with implants: A prospective trial with 13 years median follow-up in 216 patients. (2008) Eur J Surg Oncol 34(2): 143-148.

18. Mallon, P., Feron, J.G., Couturaud, B., et al. The Role of Nipple-Sparing Mastectomy in Breast Cancer: A Comprehensive Review of the Literature. (2013) Plast Reconstr Surg 131(5): 969-984.

19. Kroll, S.S., Khoo, A., Singletary, S.E., et al. Local recurrence risk after skin-sparing and conventional mastectomy: a 6-year follow-up. (1999) Plast Reconstr Surg 104(2): 421-425.

20. Kinoshita, S., Nojima, K., Takeishi, M., et al. Retrospective Comparison of Non-Skin-Sparing Mastectomy and Skin-Sparing Mastectomy with Immediate Breast Reconstruction. (2011) Int J Surg Oncol 2011: 1-7.

21. Sakurai, T., Zhang, N., Suzuma, T., et al. Long-term follow-up of nipple-sparing mastectomy without radiotherapy: a single center study at a Japanese institution. (2013) Med Oncol 30(1): 481.
Ommega Online Publishers

Journal Title: International Journal of Cancer and Oncology (IJCO)

Journal Short Name: Int J Cancer Oncol
Journal ISSN: 2377-0902

E-mail: cancerandoncology@ommegaonline.com

Website: www.ommegaonline.org 